\title{
BMJ Open Testing a model of facilitated reflection on network feedback: a mixed method study on integration of rural mental healthcare services for older people
}

Jeffrey Fuller, ${ }^{1}$ Candice Oster, ${ }^{1}$ Eimear Muir Cochrane, ${ }^{1}$ Suzanne Dawson, ${ }^{1}$ Sharon Lawn, ${ }^{2}$ Julie Henderson, ${ }^{1}$ Deb O'Kane, ${ }^{1}$ Adam Gerace, ${ }^{1}$ Ruth McPhail, ${ }^{3}$ Deb Sparkes, ${ }^{4}$ Michelle Fuller, ${ }^{5}$ Richard L Reed ${ }^{2}$

To cite: Fuller J, Oster C, Muir Cochrane $\mathrm{E}$, et al. Testing a model of facilitated reflection on network feedback: a mixed method study on integration of rural mental healthcare services for older people. BMJ Open 2015;5:e008593.

doi:10.1136/bmjopen-2015008593

- Prepublication history and additional material is available. To view please visit the journal (http://dx.doi.org/ 10.1136/bmjopen-2015008593)

Received 24 April 2015 Revised 1 September 2015 Accepted 20 October 2015

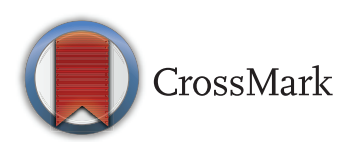

For numbered affiliations see end of article.

Correspondence to Professor Jeffrey Fuller; jeffrey.fuller@flinders.edu.au

\section{ABSTRACT}

Objective: To test a management model of facilitated reflection on network feedback as a means to engage services in problem solving the delivery of integrated primary mental healthcare to older people.

Design: Participatory mixed methods case study evaluating the impact of a network management model using organisational network feedback (through social network analysis, key informant interviews and policy review).

Intervention: A model of facilitated network reflection using network theory and methods.

Setting: A rural community in South Australia.

Participants: 32 staff from 24 services and 12 senior service managers from mental health, primary care and social care services.

Results: Health and social care organisations identified that they operated in clustered self-managed networks within sectors, with no overarching purposive older people's mental healthcare network. The model of facilitated reflection revealed service goal and role conflicts. These discussions helped local services to identify as a network, and begin the problem-solving communication and referral links. A Governance Group assisted this process. Barriers to integrated servicing through a network included service funding tied to performance of direct care tasks and the lack of a clear lead network administration organisation.

Conclusions: A model of facilitated reflection helped organisations to identify as a network, but revealed sensitivity about organisational roles and goals, which demonstrated that conflict should be expected. Networked servicing needed a neutral network administration organisation with cross-sectoral credibility, a mandate and the resources to monitor the network, to deal with conflict, negotiate commitment among the service managers, and provide opportunities for different sectors to meet and problem solve. This requires consistency and sustained intersectoral policies that include strategies and funding to facilitate and maintain health and social care networks in rural communities.

\section{Strengths and limitations of this study}

- A major strength of the study is the testing of a model of facilitated network reflection drawing on network theory and methods, including governance structure, linkage strategies and enablers, and network analysis.

- Health and social care organisations were able to see their linkages and identify as a network thereby identifying the need for a network administration organisation as the network manager with credibility and a mandate across health and social care sectors.

- Facilitated reflection on network data revealed differences between organisation and network goals and roles, which demonstrated that conflict should be expected as a part of the network manager's role.

- A lack of consistent government policy authorisation and programme resources limited the development of a network administration organisation, which was needed to maintain further cycles of facilitated reflection.

- In addition to the limitation of generalisability by focusing on only one region in South Australia, the emphasis on the service providers' perspectives of the network is a limitation. This could be addressed in future studies by basing data collection first on the consumers' help-seeking experiences, and by the inclusion of a consumer organisation in the participatory governance group.

\section{BACKGROUND}

Many individuals over 65 years will experience multiple chronic mental and physical health conditions. ${ }^{12}$ Poorer health outcomes and unmet needs for mental healthcare are reported for older people outside major cities, where $36 \%$ of the Australian population aged 65 years and over are living. ${ }^{3}{ }^{4} \mathrm{An}$ older person with a mental health problem 
may require input from a range of services across sectors. In Australia, health, aged care and social services have varied funding (public and private), governing structures (split across national, state/territory and local governments), and service scope and boundaries. These services also have different institutional and professional cultures. ${ }^{5}$ This context can be challenging for health and social care professionals and consumers to navigate, leading to poor access to optimal care. Consequences of a lack of adequate primary mental healthcare for older people include frequent and longer acute hospitalisation, deterioration of physical and mental health, and earlier admission to residential care. ${ }^{67}$

\section{A network approach}

The complexity of mental healthcare for older people in rural regions requires locally tailored and flexible services in order to respond to health and social care needs. As each community has different providers of services, developing networks to comprehensively meet the needs of older people is essential. Networks collectively use information (feedback) and organisational learning (iterative problem solving) in order to provide tailormade local solutions. However, these require management approaches that will maximise stakeholder engagement. $^{89}$

In 2013, the researchers were approached by organisations involved in mental healthcare and support of older people in a rural region in South Australia. They were being driven by national and state health policy to work in more networked ways, but were uncertain about how this was to be practically developed and managed. The organisations were the state government rural mental health service, the regional primary healthcare planning organisation funded by the national government (called Medicare Local), and an umbrella network of older people's health and social care organisations that was auspiced by the local government (called the Positive Ageing Taskforce (PATF)). In response, a model of network engagement and management entitled facilitated reflection on network feedback was developed to help rural communities improve mental health service integration. In this paper, we report on the case study in which we tested facilitated reflection as a network management process.

\section{Facilitated reflection on network feedback}

Networks are built on the collaborative relationships between individuals and organisations with trust and mutual exchange as the core to their success. ${ }^{10}$ For networks to function effectively they require cooperation and commitment between participating organisations that are distinct and often autonomous, ${ }^{11}$ and as such these networks pose different management challenges to the more traditional forms of service organisations, such as bureaucratic hierarchies and markets. ${ }^{12-14}$ A key role for network managers and leaders is to 'establish a foundation on which network participants can operate' (ref. 9, p.40). Modes of leadership that rely on the role of facilitator/broker are necessary, with the overarching management role being to 'increase the stock of trust and reciprocity in the network' (ref. 9, p.46).

The complexity and idiosyncratic nature of networks means that research findings about network functioning can be difficult to generalise into standardised and prescribed actions. ${ }^{15}{ }^{16}$ Instead, reflective practice is proposed as a method to help participating organisations develop collaboratively into a network and then, over time, to improve the network functioning. This approach involves stimulating network members to reflect on their collective goals, the problems they face, the resources that they have, and then the actions they can take that will work for them. ${ }^{15}{ }^{16}$ Hibbert $e t a l^{15}$ see the development of 'handles for reflective practice' (p. 405), in which partners formulate their actions in light of their own circumstances and competencies, as the generalisable feature of network management. We propose that a key role for network management is to underscore the common goals of network members while facilitating them to reflect on their situation and work together to solve identified problems. ${ }^{12} 17$

The notion of 'facilitation' is used here in two ways: first, it refers to the use of a facilitator to support organisations as they self-evaluate and take action as a network. The notion of facilitated reflection is particularly important in the context of interorganisational human service networks, where organisations are often from different sectors, with different goals and management structures and are often competing for the same pool of resources. In the study presented here, the facilitator took the form of an external (university-based) research team, working with the three main stakeholder organisations involved in older people's mental health servicing in the case study region. Second, facilitated reflection on network feedback refers to the theories and tools used to facilitate members of human services organisations to reflect on their own unique collective situation and experiences. We derived these notions from the fields of interorganisational relations and complexity theory, ${ }^{13}$ and drew on three aspects of network theory and methods-namely, governance structure, linkage strategies and enablers and network analysis. These approaches were used to develop a model for facilitated reflection on network feedback tested in this study. The model process responds to Vangen and Huxham "handles for reflective practice" that "aim to support participants in understanding their collaborative relationships and so allow them to devise their own management strategies" (ref. 13, p. 757).

\section{Governance structure}

Exploring the governance structure operating in a network, and determining which is ideal to meet local needs is an important task for network management. ${ }^{9}$ Kenis and Provan ${ }^{18}$ have described three structural models of network governance against which they place two dimensions of brokerage and participation. At one 
extreme, a self-governing network will have all members equally participating in governance tasks. At the other extreme, one agent functions as the broker who governs the network. The three models are illustrated in figure 1 below.

Governance structures often evolve over time and in practice, governance models are frequently a hybrid of one or more of these three types. ${ }^{9}$ Kenis and Provan ${ }^{18}$ suggest that brokered governance structures are more effective where there is a lower level of trust across the network, higher number of participants, lower goal consensus and increased need for network level competencies.

\section{Linkage strategies and enablers}

A network approach to servicing requires the use of specific linkage strategies for primary mental healthcare services to work together, ${ }^{19}$ as well as the presence of enablers who support these linkage strategies. ${ }^{17}$ An exploration of the extent to which these linkage strategies and enablers are present in a network provides a description of network function, capacity and areas for improvement, particularly through the use of a continuous cycle of evaluation feedback. ${ }^{20} 21$

\section{Network analysis}

Network analysis is a quantitative method of data collection and analysis used to examine the links through which individuals and organisations interact with one another. Network data can be presented as a picture of the number and strength of links (relationships) between organisations, as reported by members of the organisations. ${ }^{22}$ Network analysis can explore the structure of interorganisational relationships to provide information about what the network looks like. This information can be used with other qualitative data to assist managers in building and sustaining local networks. $^{23} 24$

\section{METHODS}

We conducted a mixed methods case study over 18 months (2013-2015), with a complete description of the methods previously published. ${ }^{25}$ We report on the following two study questions:
1. What organisational links related to mental healthcare for older people currently exist, and what are the enablers and barriers to developing these (network servicing)?

2. How effective is a model of facilitated reflection that uses network feedback to manage networked primary mental healthcare for older people?

Figure 2 displays one cycle of facilitated reflection on network feedback. The intent was that one or more of the stakeholder organisations would take over and continue the cycles, reflecting the recursive relationship between network management and network development identified by Sydow. ${ }^{11}$

Expected outcomes were to:

- Establish network commitment for older people's mental healthcare in the region. For the purposes of this study, commitment was defined as ongoing participation and engagement of study participants throughout the project and the formulation of an agreement among the three stakeholder organisations to continue meeting and working together following the conclusion of the project;

- Develop agreed protocols;

- Improve service coordination

\section{Setting}

The setting was the southern part of the Adelaide Hills, Fleurieu and Kangaroo Island Medicare Local region in South Australia. The economy of the region is largely agricultural and tourism based, and it has experienced a $52 \%$ increase in those aged 65 years and over from 2001 to $2011 .^{26}$ While relatively well serviced, it is typical of many Australian rural locations that are within $100 \mathrm{~km}$ of metropolitan centres, but which still face difficulties in specialist service access, coordination and follow-up because of differences in funding criteria and boundaries between services.

\section{Data collection}

In order to generate network data for facilitated reflection, the data collection involved: (1) a quantitative organisational network analysis, (2) qualitative interviews with service provider key informants and consumers or their carers and (3) a policy document analysis. As the data from the consumer/carer interviews informed a separate

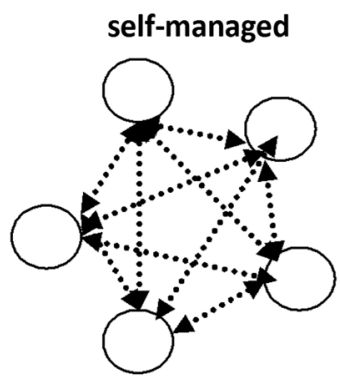

lead organisation

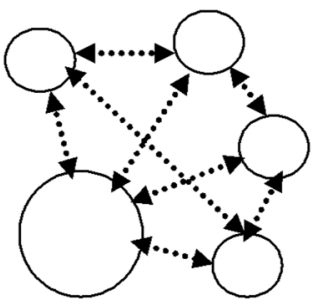

network admin organisation

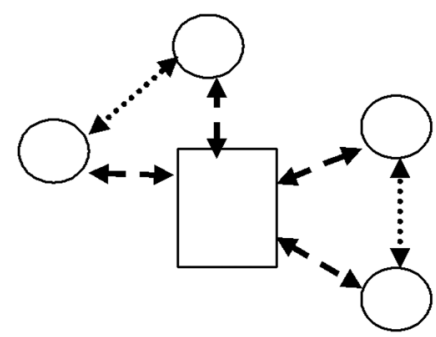

Figure 1 Typology of network governance. ${ }^{18}$ 


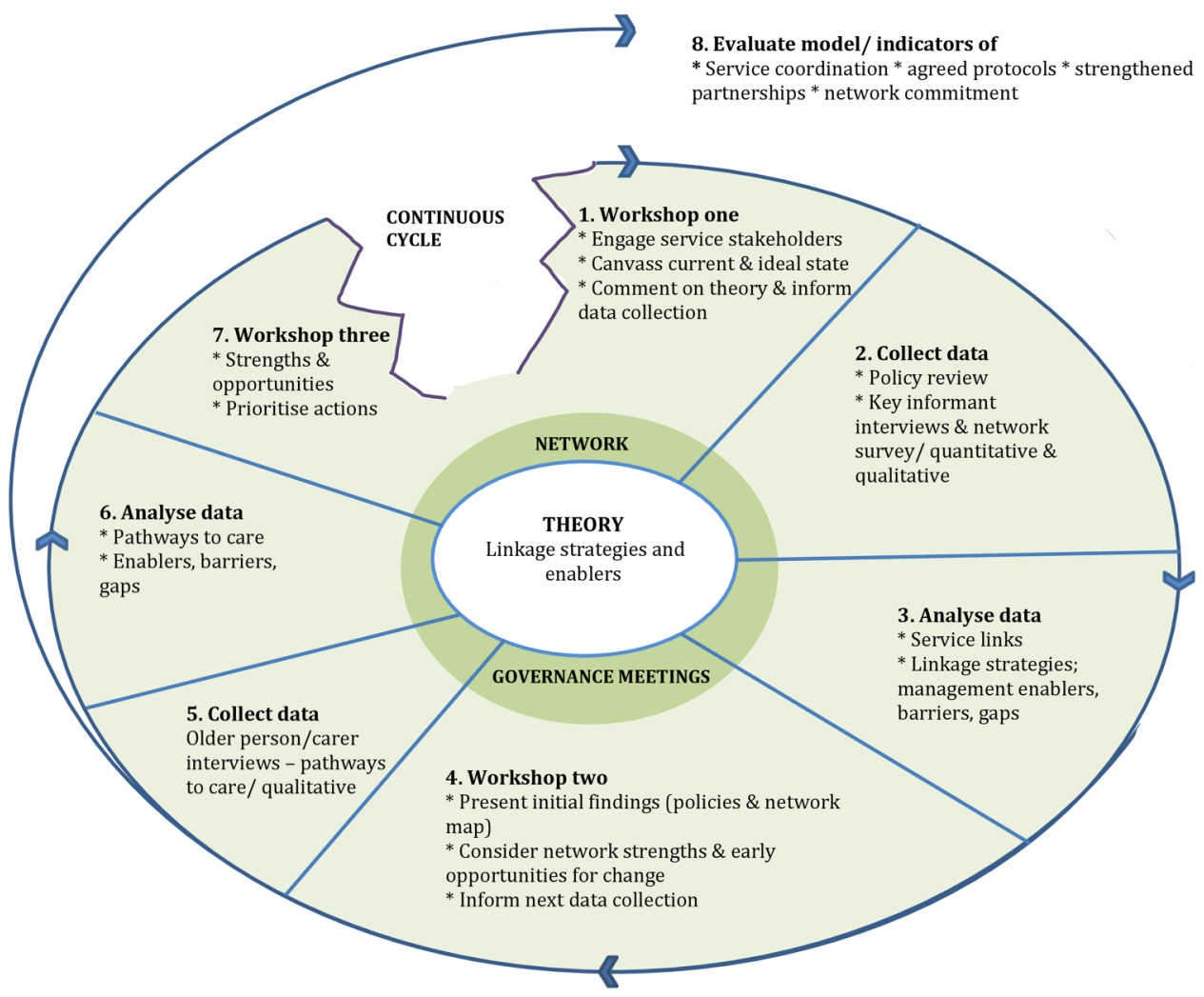

Figure 2 Diagram of facilitated network reflection cycle.

research question related to service gaps and opportunities, these data are not reported in this paper, but are noted as the interviews did provide material for feedback and reflection on these gaps and opportunities.

\section{Participants and process}

Governance Group

A Governance Group was established comprising three senior staff members with management responsibilities in the main stakeholder organisations related to older people's mental health in the region. Seven Governance Group meetings occurred over the 18 months of the project with minutes from these meetings included as data.

\section{Service provider key informant interviews}

Participants for service provider key informant interviews were recruited via purposive sampling based first on suggestions made by the Governance Group and then by participants in workshop one. Key organisations and staff providing mental health and related services to older people in the region were identified across mental health $(n=3)$, primary healthcare $(n=13)$, and aged care and social service organisations $(n=8)$. All informants approached by the researchers agreed to participate except for one GP, but we were able to interview the nurse in that practice. We conducted individual and group interviews with 32 key informants from 24 organisations. The interviews were semistructured using a quantitative organisational network analysis tool ${ }^{2327}$ and open-ended qualitative questions. This enabled us to examine the regional service network structure and to gain service provider perspectives on the linkage enablers and the barriers between them.

The network survey asked participants to list up to 15 services with whom they (and others in their organisation) had communicated over the past 3 months, focusing on services they considered to be the most important in their organisation for providing care and support for older people with mental health issues. Participants were asked to score each listed service as per the number of times they communicated with the service over the past 3 months $(1=$ less than once per week; $2=$ about once per week; $3=$ more than once per week) about client care (information/referral) and the management, planning, and operation of services for mental healthcare for older people in the region.

Qualitative interviews included questions about processes that support communication links with other services, operational or procedural policies that inform how they work with other services, examples of services working well together and those not working well together, and anything they would like to see changed in the way services work together to meet the needs of older people with mental health issues.

As a result of a feedback loop, later interviews were also conducted with 12 senior managers from the major service providers (mental health $=1$, primary healthcare $=7$ and aged and social care $=4$ ) after the second workshop in order to gain a management perspective on the servicing and networking issues. These senior 
manager interviews included additional questions about leadership and management strategies, and feedback on the mid-project report.

\section{Policy review}

Policy documents at the national and state government levels $(n=22)$, and the local services operational level $(n=24)$ were examined for relevance to networked mental health servicing for older people.

\section{Workshops}

Workshop participants were recruited initially according to the recommendation of the Governance Group and then from among participants in the key informant interviews. Participants included workers and senior leaders from mental health, primary care, aged care, social care and consumer organisations across the region. Findings were presented to participants progressively at each workshop, where facilitated group reflection was used to identify network issues regarding older people's mental health servicing and potential solutions. In workshop one, participants $(n=12)$ were asked to reflect on three case scenarios in relation to: (1) the linkage strategies already in place in their region, and (2) ideal linkage strategies to address the problems presented in the scenarios. Workshop two $(n=18)$ involved reflection on the findings from the service provider key informant interviews, focusing on current service links, enablers and barriers to effective linkages and the early available opportunities for change. In the final workshop, findings from the consumer/carer interviews and the senior manager interviews were presented and participants $(n=17)$ were asked to identify opportunities for change, assign a value analysis (importance and 'do-ability') to the opportunities and develop a plan of action for high-value opportunities. These opportunities reflect Sheaff $e t$ al $\mathrm{s}^{28}$ 'core artefacts' by focusing on what products the network should produce, rather than on trying to resolve differences about member values and goals. The production of core artefacts drives the development of a network's culture through which reciprocity and trust can grow, which has been identified as a key task of network management. ${ }^{28}$

\section{Knowledge exchange}

Knowledge exchange activities were undertaken to facilitate ongoing engagement and ownership. These included engaging the main service stakeholders in the planning and ongoing implementation of the project through the Governance Group, distributing summary reports to participants, presentation of a mid-project report at various forums and meetings in the region, and maintaining a project blog.

\section{End of project interviews}

Further interviews were conducted at the end of the project with five senior leaders from the three stakeholder organisation involved in the Governance Group to explore whether they had found the facilitated reflection process useful or not, and the sustainability of this process.

\section{Data analysis}

Organisational network analysis was used to quantitatively examine which organisations were linked, the number of links in the network and the types of linkage interactions between organisations (eg, exchanging information, referrals and planning). ${ }^{23}{ }^{27}$ Maps and tables displaying the patterns of connections between organisations were generated using the UCINET software. ${ }^{29}$

Framework analysis $^{30}$ was used with the qualitative interview data from the service provider key informants and the workshop discussions to describe the linkage strategies, and linkage enablers and barriers between services. Policy documents were examined to explore how the issue of mental health and older people is described and addressed, and the implications of this for the development of networked servicing in the region. We also analysed the minutes of the Governance Group, the records of the service provider workshops, and the end of project interviews to evaluate the effectiveness of facilitated reflection.

\section{RESULTS}

\section{Networked servicing}

Information about the current state of the interoganisational linkages, including the linkage strategies and enablers that were present in the region, was provided to participants over the course of the project through the three workshops. At the first workshop it was identified that the services work more together within the three subregions than they do across the region as a whole, and so maps were constructed for each of these three subregions. The network analysis of the 24 surveyed organisations across the three subregions revealed that organisations link more frequently with others within their sector than they do between sectors. This can be seen in the following network map on the function of service management, planning and operations in the largest subregion (figure 3). Black nodes represent healthcare organisations, hashed nodes are aged and social care organisations, and white nodes are other nominated linked organisations that were not surveyed. Nodes with similar patterns of links are placed in closer proximity (clustered) and thicker lines indicate more frequent linking. Only 14 of the surveyed services in this subregion ( 7 health, and 7 aged and social care) indicated that they linked with other organisations on this function. They nominated an additional 23 organisations with whom they link but we did not survey these organisations. Two of these non-surveyed organisations were well linked on this function (a medical practice and a home care assessment service), thereby indicating 
Figure 3 Network services reporting links to do with service management, planning and operations (main subregional location).

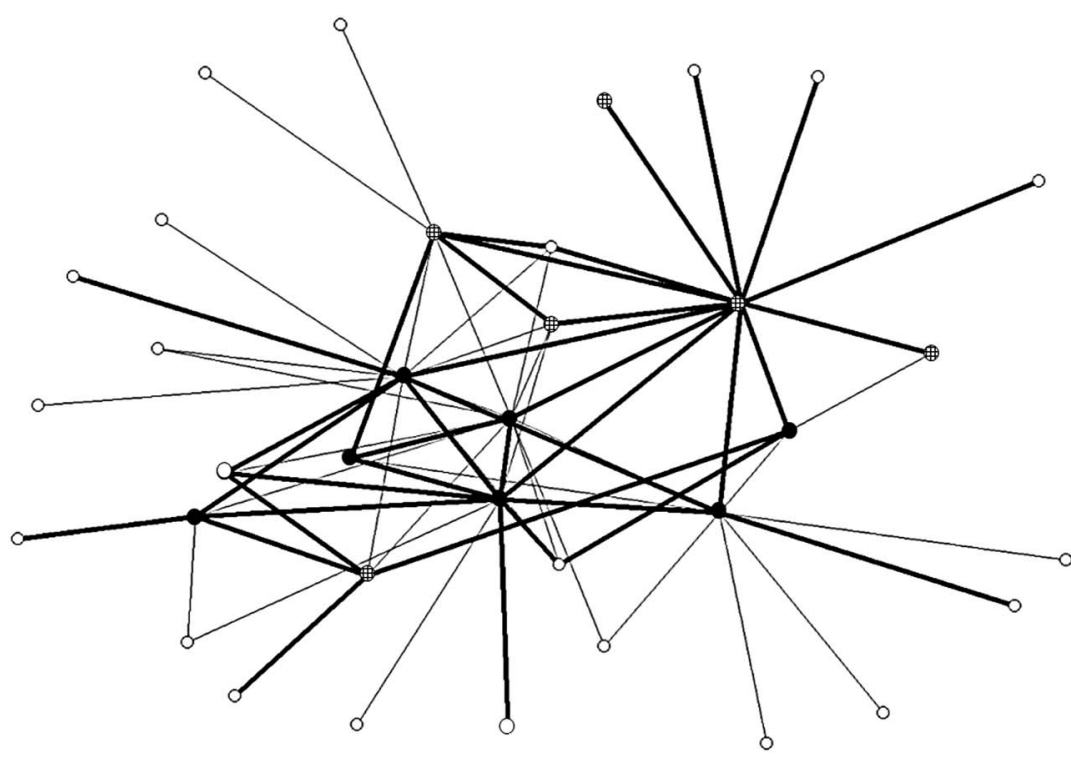

these as possible important services to include in mental health network development.

Across the whole region (three subregions combined), 19 of the 24 surveyed services indicated that they link with 69 other organisations on the function of service management, planning and operations with a total of 174 link ties. The main nominated organisation that was linked to on this function (highest in-degree centrality of 8) was the Medicare Local (probably due to its regional planning function). We concluded from this that the pattern of links did not identify a clear organisation with a mandate to take on overall management and governance functions for a network of older people's mental health servicing nor one that covered the broader range of health and social care. Discussion at the second and third workshops, and the later interviews with service managers canvassed this as either a role for the Medicare Local (or its successor) or the PATF. Hence, participants concluded that at present there was no single formal purposive network that covered older people's mental health servicing, as the following GP described:

there's a severe lack of coordination and communication at all levels. We've got too many organisations trying to provide the same services ...there's nobody coordinating or getting all these groups together to see if we can coordinate things ... we're not talking to each other and there's duplication of services and it's just so frustrating.

Instead there appeared to be various groupings of services that linked for different purposes and functions. When exploring in workshop two the linkage strategies that were present in the region, we identified that services were connected through a range of meetings, with varying degrees of formality and service commitment. The smaller groupings were typically localised and clustered by same service type rather than across the health and social care sectors as a whole. For instance, it was reported that the weekly multidisciplinary meetings at a remote location worked well because the meeting had a focused function, the location had a small population, staff knew each other, and they had an identification and commitment to this location. In comparison, the larger regional grouping of the PATF was not highly linked to the mental health services, possibly because the Taskforce had a broad remit across a range of issues across the whole region. While the Taskforce was established to facilitate service development, planning and collaboration between services, this was not by any formal interorganisational agreement or specific to mental health. There were no active cross-sector meetings specific to older people's mental health servicing. This was highlighted in the network reflections, which led to discussion in workshop three about ways to change this through some form of service agreement.

We can conclude, therefore, that the grouping of services through various clustering of links and strategies (meetings) was only a very embryonic form in the typology of purposive goal-oriented networks. The Medical Local appeared to have a potential management and governance role as a network administration organisation to develop an older people's mental health servicing network because of higher centrality on this function, and because of its remit as a regional healthplanning organisation. Until the third workshop, however, there was no agreement, authorisation or resources for a network administration organisation and with the later cessation of Medicare Local funding, this potential role was not able to progress.

In the analysis of strategies and enablers, linkage strategies appeared to be involved more with direct collaborative activities, the various use of guidelines and some communication systems rather than with formal agreements (see table 1). While this was effective in smaller locations in the region, informants explained that 
Table 1 Linkage strategies

\begin{tabular}{|c|c|c|c|}
\hline Category & Strategy & Present & Comments \\
\hline \multirow{4}{*}{$\begin{array}{l}\text { Direct collaborative } \\
\text { activities }\end{array}$} & Link working & $\checkmark \checkmark$ & Predominantly informal links and localised, some OPMH specific \\
\hline & Co-location & $\checkmark \checkmark$ & $\begin{array}{l}\text { Various services co-located across the region, mainly health and } \\
\text { some aged care services }\end{array}$ \\
\hline & Consultation liaison & $\checkmark \checkmark$ & $\begin{array}{l}\text { OPMHS role (local) and psychiatric and geriatrician review } \\
\text { (visiting) but GPs and social care services looking for support }\end{array}$ \\
\hline & Care management & $\checkmark \checkmark$ & $\begin{array}{l}\text { Good within and across services, some gaps when the consumer } \\
\text { has needs that cross health and social care-no overarching } \\
\text { cross-sector coordination }\end{array}$ \\
\hline \multirow[t]{2}{*}{ Agreed guidelines } & Protocols & $\checkmark$ & $\begin{array}{l}\text { Protocols for general mental health-none OPMH specific that } \\
\text { cross health and social care }\end{array}$ \\
\hline & Stepped care & $\checkmark \checkmark$ & Predominantly between health and aged care \\
\hline \multirow[t]{3}{*}{$\begin{array}{l}\text { Communication } \\
\text { systems }\end{array}$} & $\begin{array}{l}\text { Enhanced } \\
\text { communication }\end{array}$ & $\checkmark \checkmark$ & $\begin{array}{l}\text { Various meetings in the region but no overarching formal } \\
\text { mechanism with commitment from health and social care; } \\
\text { Informal mechanism working well in small subregion }\end{array}$ \\
\hline & Enhanced referral & $\checkmark \checkmark$ & $\begin{array}{l}\text { Range of formal and informal process in place but some lack of } \\
\text { knowledge about these across services in the region }\end{array}$ \\
\hline & $\begin{array}{l}\text { Electronic } \\
\text { communication system }\end{array}$ & $\checkmark$ & Limited to health services \\
\hline Service agreement & Service agreement & $\checkmark$ & $\begin{array}{l}\text { No specific agreements for OPMH other than more general MOU } \\
\text { between police, SAAS and mental health. }\end{array}$ \\
\hline \multicolumn{4}{|c|}{$\begin{array}{l}\text { Criteria: Older people's mental health (OPMH) specific; regional as compared to localised; and formal and regular compared to ad hoc } \\
\text { No criteria evident }(\mathrm{X}) \text { through to all three criteria evident }(\checkmark \checkmark \checkmark) \text {. } \\
\text { For full definition of linkage strategies see Fuller et al. }{ }^{19} \\
\text { GPs, general practitioners; MOU, memorandum of understanding; OPMHS, Older Person's Mental Health Service; SAAS, South Australian } \\
\text { Ambulance Service. }\end{array}$} \\
\hline
\end{tabular}

relying on direct personal relationships and informality was less effective in the larger locations with greater complexity in servicing.

Enablers to organisations linking in the region were generally related to partnership formation activities undertaken by workers engaged in service delivery, and by workers who were committed to doing this. There did not appear to be a higher level government policy or programme support related to older people's mental health or cross-sector authorisation or resources for networking; but rather funding tied to performance of direct care tasks (see table 2).

When bringing together a range of services, sensitivities and conflicts are likely to become evident. ${ }^{12}$ Two such sensitivities did arise. The first related to whether sufficient assistance was being provided to enable organisations to optimally refer clients. The second related to how the network maps described which organisation was the most central in the development of mental health services for older people. Both issues were successfully resolved through discussion and advice in the Governance Group.

\section{The effectiveness of facilitated reflection}

By reflecting on the range of issues identified through the feedback of network data, facilitated reflection aimed to support organisations to work together as a network in order to solve problems related to community mental healthcare for older people. The effectiveness of the process is presented against the expected outcomes.

\section{Establish network commitment}

The processes used to establish network commitment included workshops and knowledge exchange. Face-to-face contact at the workshops gave participants from different services the opportunity to gain a broader understanding of older people's mental health servicing in the region, and to clarify issues that were important across services. As a part of the feedback from the Governance Group and workshop two, we realised that higher-level leadership input from some of the main organisations across the sectors was missing. It was for this reason that we conducted targeted interviews with 12 service managers and thus secured participation from three more senior managers at workshop three. At the third workshop, participants established priority actions about care pathways; cross-sector training and referral; the need for a service agreement, expressed particularly by the mental health service senior manager; and inclusion of older people's mental health in the local public health plan. The partner stakeholders highlighted the value of the three workshops in establishing network commitment through raising awareness and creating excitement for change:

it's been useful getting everyone together ...that's increased the understanding of everyone of all ... [the] services that are available and so obviously better communication, and the feedback plus the opportunities and learning about referrals and things ... the excitement that things can be improved and what possibilities there were. (Health Service) 
Table 2 Linkage enablers

\begin{tabular}{|c|c|c|c|}
\hline Category & Enabler & Present & Comments \\
\hline $\begin{array}{l}\text { Government policy and } \\
\text { programme support }\end{array}$ & & $x$ & $\begin{array}{l}\text { Integration advocated but lack of implementation } \\
\text { strategy; No detail regarding older people's } \\
\text { mental health }\end{array}$ \\
\hline \multirow[t]{3}{*}{$\begin{array}{l}\text { Organisational level: } \\
\text { leadership }\end{array}$} & Authorise/encourage team work & $\checkmark$ & $\begin{array}{l}\text { Authorisation around specific issues but limited } \\
\text { meetings to enable teamwork; Concern } \\
\text { re-permissions to attend meetings }\end{array}$ \\
\hline & Resources and strategies & $\checkmark$ & $\begin{array}{l}\text { Limited resources and formal strategy; Dedicated } \\
\text { staff in OPMHS clinician, PATF project officer, } \\
\text { SAFKI rural coordinator; Service funding tied to } \\
\text { occasions of direct care }\end{array}$ \\
\hline & Recruitment and staff development & $\checkmark \checkmark$ & $\begin{array}{l}\text { As above recruitment into staff positions-MHFA } \\
\text { training }\end{array}$ \\
\hline $\begin{array}{l}\text { Worker level } \\
\text { partnership formation } \\
\text { activities }\end{array}$ & $\begin{array}{l}\text { Joint development through active } \\
\text { communication; } \\
\text { Share information, plan and problem } \\
\text { solve }\end{array}$ & $\checkmark \checkmark$ & $\begin{array}{l}\text { As the above (table 1) various meetings-works } \\
\text { well informally in small subregion with long-term } \\
\text { workers embedded in the community; Larger } \\
\text { subregion needing more formalised processes }\end{array}$ \\
\hline $\begin{array}{l}\text { Worker attribute } \\
\text { enabler }\end{array}$ & $\begin{array}{l}\text { Commitment to collaboration; } \\
\text { Skills in primary care and mental } \\
\text { health; Primary care focused and } \\
\text { flexible work style; } \\
\text { Ability to fit into teams }\end{array}$ & $\checkmark \checkmark$ & $\begin{array}{l}\text { As above-committed workers discussed in } \\
\text { service provider and consumer/carer interviews- } \\
\text { expressed limits on participation in meetings }\end{array}$ \\
\hline $\begin{array}{l}\text { Evaluation and } \\
\text { feedback }\end{array}$ & & $\checkmark$ & $\begin{array}{l}\text { Limited but with some via the PATF } \\
\text { communicating with the members }\end{array}$ \\
\hline Proximate location & Interaction capacity & $\checkmark \checkmark$ & $\begin{array}{l}\text { Evidence of this working well in the small } \\
\text { subregion and in some centres }\end{array}$ \\
\hline
\end{tabular}

Commitment to the network was demonstrated by the ongoing participation and engagement of service workers throughout the project, of which 11 from the 12 attending the first workshop participated in the subsequent workshops, in the service stakeholder interviews and in the recruitment of consumers and carers for interviews.

Commitment to the network from management was demonstrated by an agreement at the conclusion of the final workshop for the three stakeholder organisations in the Governance Group to meet and work together on the plan for priority actions mentioned above. This was despite the funding that was to cease for two of the main stakeholder organisations (Medicare Local and PATF) during 2015.

\section{Development of agreed protocols}

The facilitated reflection led to some direct actions being taken to solve identified network problems. In workshop two, the analysis of service linkages identified gaps in communication between general practitioners (GPs) and the aged and social care providers. This resulted in the development of a referral template, and the worker who facilitated this presented the template to participants at workshop three. Lack of knowledge of service options (highlighted in service provider key informant interviews) led to plans to develop a directory of social care services to be 'housed' on the local government website. Each of these actions involved workers from different services collaborating and thereby, further developing links within the network.

\section{Improved service coordination}

Despite the achievements discussed above, improved service coordination was not realised between workshops one and three. This was probably due to the network complexities for different service criteria and misunderstanding about roles that can increase the time required for interorganisational change. ${ }^{31-33}$ This complexity was compounded in this case by what informants noted as the current funding uncertainty for services in older people's mental health, the uncertainty in service contracts to non-government organisations and also in the organisational changes that were occurring in the Medicare Local, whose functions were being transitioned to a new organisation (called Primary Health Networks) by the national government. This revealed an obvious leadership hiatus when it was evident in the management and final stakeholder interviews that a dedicated network administration organisation was required to facilitate and maintain the process of network reflection. 


\section{DISCUSSION}

In order to best meet the complex care needs of older people with mental health problems, services would benefit from considering themselves as part of a network to better use resources, improve care coordination and ensure capacity building in the workforce and in the broader community. ${ }^{9}$ Yet networks are inherently problematic from a management perspective due to the need to bring together distinct organisations, often with different cultures and goals ${ }^{12} 34$ and a lack of management power and control. ${ }^{14}$ The aim of this study was to explore the effectiveness of a management model of facilitated reflection on network feedback through which organisations can work together as a network to meet the mental healthcare needs of older people.

Drawing on Hibbert et $a l \mathrm{~s}^{15}$ discussion of reflective practice as the generalisable feature of network management, we developed a process of facilitated reflection based on network theory and methods. By reflecting on governance structure, ${ }^{19}$ linkage strategies and enablers, ${ }^{17} 19$ and data from a network analysis ${ }^{23}$ facilitated through workshops and Governance Group meetings, the process started to build connections. Network analysis and mapping helped local services to see their pattern of links and identify problems in interagency communication and referral. Workshops established a range of priority actions and facilitated partnerships with the development of the referral template engaging GPs, aged care services and the Medicare Local. However, due to the lack of a network administration organisation and the end of funding for both the Medicare Local and PATF in 2015 (both having some network management functions), the network development and momentum remained fragile.

It was clear that one cycle of facilitated network reflection (which was determined by the project funding) was insufficient to cement improved service coordination and sustain the change, as the literature on interorganisational networks indicates. ${ }^{31-33}$ The use of ongoing cycles of facilitated reflection is, therefore, recommended. ${ }^{112023}$ Our study has focused on measurement of network structure and used qualitative data to establish degree of commitment, protocol development and coordination. Future cycles could include further network surveys to see how the pattern of linkages changes, as well as study other tools that are used more effectively to empirically measure network characteristics such as commitment, ownership and trust. ${ }^{35-37}$

The study demonstrated the need for a network administration organisation in the region with the mandate to coordinate services and with the relevant expertise to address specific issues. For network facilitation to be effective, a network administration organisation needs to be seen to be neutral and have credibility across health and social care, with management and leadership oriented towards building trust and reciprocity through facilitation and brokerage. ${ }^{12}$ 'Unobtrusive leadership' is important so that network members do not see the network administration organisation as controlling and interfering in their individual internal issues. ${ }^{12}$ That said, management and leadership should not be 'soft' in the willingness to continually renegotiate organisational commitment to the network, and also not be put off by the conflicts around goals and roles that will invariably occur. ${ }^{14}$

\section{Limitations}

Limitations of the study include methodological issues associated with collecting social network data about organisations, such as the lack of generalisability to other service networks, the potential bias in how network membership and hence the network boundary is determined and the impact of missing links when some nominated organisations are not surveyed. ${ }^{24}$ The generalisable application of the method that we have described in this one rural region does need a consideration of the impact of rurality on how services work together, and what might differ if this method were to be used in a different region. The region in this study comprised three subregions, one that was considered remote and where linking across services appeared to work without formal agreements, compared to what might be needed in larger locations and for sustainability, as we found in another study. ${ }^{38}$ Owing to the constraints of service funding tied to occasions of service (see table 2), rural regions may find the use of network reflection logistically and resource difficult because they have less staff available to travel greater distances and therefore, they would need to spend more time away from service delivery to undertake the reflective process.

A further limitation relates to our confidence that the individuals surveyed could adequately capture the interorganisational links without the maps being a biased representation. ${ }^{23}$ While we cannot be certain that the maps represent the real structure of organisational linkages, we did seek to recruit participants with organisational knowledge. On the initial advice of the Governance Group, and further advice from workshop one, we specifically recruited participants considered to be knowledgeable because of their position or length of time in their job. Furthermore, at workshop two we had participants reflect on whether the maps conveyed their experience of the linkages, and whether organisations or links were missing. In the main, the maps appeared to be adequate, particularly as the intent was that these be used as a heuristic device rather than as a set of empirically accurate measurements. ${ }^{39}$

The most obvious bias related to some missing GP input into the survey, as many GPs operate as sole practitioners even when they are in a group practice. We could not interview every GP and we knew that GPs were difficult to recruit into studies. ${ }^{40}$ Hence, we took the pragmatic approach to collectively consider general practice (not the GP) as a network node by surveying the main practice known to see patients with a mental illness in a location. There were only two locations 
where this was relevant and where we did not survey all of the practices. We also accepted either the GP or the nurse working in that practice as the interviewee, and so it is reasonable to conclude that the links may have been reported differently depending on who was interviewed.

A final limitation of the study is that it did not develop a more consumer-focused approach to the facilitated reflection process, as recommended in recent research on integrated care. ${ }^{41}{ }^{42}$ As our intent was to trial the reflective process as a means for managers to improve service networking, we considered indicators of success as those related to network processes (commitment, protocols and coordination), and not the outcomes that might come from this, such as improved consumer experience or some indicator of service quality. To better include the consumer perspective we think the method could be refined in the following ways. First, mapping of consumer care seeking journeys could form the basis of the list of services for the organisational network analysis. In this study, the Governance Group provided this framework, which meant that the organisational network analysis revealed the network more from the service provider perspective than from the perspective of the consumer and carer. Second, having a consumer organisation included on the Governance Group would have been helpful when dealing with network conflicts, as the consumers' need would then be reinforced as the goal to be held in common by the network members.

\section{CONCLUSION}

The case study provides valuable information about the effectiveness of a model of facilitated reflection on network feedback as a network management process in the context of rural mental healthcare for older people. We found that facilitated reflection helped local services to identify as a network and to begin problem-solving of interagency communication and referral links. The process does take up resources and time, which must be factored in by policy and funders, particularly as there is a need for a network administration organisation to continue the process of network reflection. The findings from this study are applicable to other contexts where interorganisational networks are established to address complex healthcare needs.

\section{Author affiliations \\ ${ }^{1}$ School of Nursing \& Midwifery, Flinders University, Adelaide, South Australia, Australia \\ ${ }^{2}$ School of Medicine, Flinders University, Adelaide, South Australia, Australia ${ }^{3}$ Country Health South Australia Local Health Network Mental Health Services, Adelaide, South Australia, Australia \\ ${ }^{4}$ Southern Adelaide-Fleurieu-Kangaroo Island Medicare Local, Adelaide, South Australia, Australia \\ ${ }^{5}$ City of Victor Harbor, Victor Harbour, South Australia, Australia}

Contributors JF, CO and EMC designed the study. DO, JH, SL, AG and RR provided methodological input to the study design. RM, DS and MF provided input for the feasibility of the study, and stakeholder engagement and data collection processes. CO and SD undertook the literature review. JF, CO, EMC,
$\mathrm{DO}, \mathrm{JH}, \mathrm{AG}$ and SD contributed to the data collection and analysis. All authors contributed to the writing of the paper and all have approved the final version.

Funding The research reported in this paper is a project of the Australian Primary Health Care Research Institute, which is supported by a grant from the Commonwealth of Australia as represented by the Department of Health and Ageing. The information and opinions contained in it do not necessarily reflect the views or policy of the Australian Primary Health Care Research Institute or the Commonwealth of Australia (or the Department of Health and Ageing). Additional funding was also provided by a grant from the Nurses' Memorial Centre of South Australia and the Faculty of Medicine, Nursing and Health Sciences, Flinders University of South Australia.

Competing interests At the time of the study RM, DS and MF were all employed in organisations that were part of the service network that is the focus of this study.

Ethics approval The Human Research Ethics Committees of the South Australian Health Department (HREC/13/SAH/126) and Flinders University (notification10/2014)

Provenance and peer review Not commissioned; externally peer reviewed.

Data sharing statement The raw data are securely stored in a password-protected server at the Flinders University of South Australia and made available to the research team only. In line with the participatory nature of the research design, analysed data in a de-identified form will be made available to participants. Hence, these data along with the reporting to the funder will be available to other researchers on application to the corresponding author.

Open Access This is an Open Access article distributed in accordance with the Creative Commons Attribution Non Commercial (CC BY-NC 4.0) license, which permits others to distribute, remix, adapt, build upon this work noncommercially, and license their derivative works on different terms, provided the original work is properly cited and the use is non-commercial. See: http:// creativecommons.org/licenses/by-nc/4.0/

\section{REFERENCES}

1. Glynn LG, Murphy AW, Smith SM, et al. Self-monitoring and other non-pharmacological interventions to improve the management of hypertension in primary care: a systematic review. $\mathrm{Br} J$ Gen Pract 2010;60:e476-88.

2. OECD. Health reform meeting the challenge of ageing and multiple morbidities. OECD Publishing, 2011 [cited 10 Feb 2015]. http://www. oecd.org/els/health-systems/49151107.pdf

3. Haralambous B, Xiaping L, Dow B, et al. Depression in older age: a scoping study. Australia: National Ageing Research Institute, 2009.

4. Royal Australian \& New Zealand College of Psychiatrists. Priority must be given to investment that improves the mental health of older Australians [internet]. Position Statement 71; November 2011 [cited 10 Feb 2015]. https://www.ranzcp.org/Files/Resources/College_ Statements/Position_Statements/ps71-pdf.aspx

5. Kodner DL, Spreeuwenberg C. Integrated care: meaning, logic, applications, and implications-a discussion paper. Int J Integr Care 2002;2:e12.

6. Draper B, Low L. What is the effectiveness of old-age mental health services? Health Evidence Network Report. Copenhagen: WHO Regional Office for Europe, 2004 [cited 10 Feb 2015]. http://www. euro.who.int/_data/assets/pdf_file/0008/74690/E83685.pdf

7. Ryan AA, McCann S, McKenna $\mathrm{H}$. Impact of community care in enabling older people with complex needs to remain at home. Int $J$ Older People Nurs 2009;4:22-32.

8. Ferlie E, Fitzgerald L, McGivern G, et al. Public policy networks and 'wicked problems': a nascent solution? Public Adm 2011;89:307-24.

9. Popp JK, Milward HB, MacKean G, et al. Inter-organizational networks: a review of the literature to inform practice. IBM Center for The Business of Government, 2014.

10. Provan K, Kenis P. Modes of network governance: Structure, management, and effectiveness. J Publ Adm Res Theor 2008;18:229-52.

11. Sydow J. Network development by means of network evaluation? Explorative insights from a case in the financial services industry. Hum Relat 2004;57:201-20. 
12. Vangen S, Hayes JP, Cornforth C. Governing cross-sector, inter-organizational collaborations. Public Manag Rev 2014;17:1237-60.

13. Vangen $\mathrm{S}$, Huxham $\mathrm{C}$. The tangled web: unraveling the principle of common goals in collaborations. J Public Adm Res Theory 2012;22:731-60.

14. Goodwin N. Are networks the answer to achieving integrated care? $J$ Health Serv Res Policy 2008;13:58-60.

15. Hibbert $P$, Huxman $C$, Smith Ring P. Managing collaborative inter-organizational relations. In: Cropper S, Ebers M, Huxman C, et al., eds. The Oxford handbook of inter-organizational relations. Oxford: Oxford University Press, 2010:390-416.

16. Huxham $C$, Hibbert $P$. Use matters ... and matters of use. Public Manag Rev 2010;13:273-91.

17. Fuller JD, Perkins D, Parker S, et al. Building effective service linkages in primary mental health care: a narrative review part 2 BMC Health Serv Res 2011;11:66.

18. Kenis $\mathrm{P}$, Provan K. Towards an exogenous theory of public network performance. Public Adm 2009;87:440-56.

19. Fuller JD, Perkins D, Parker S, et al. Effectiveness of service linkages in primary mental health care: a narrative review part 1. BMC Health Serv Res 2011;11:72.

20. Blasinsky M, Goldman HH, Unutzer J. Project IMPACT: a report on barriers and facilitators to sustainability. Adm Policy Ment Health 2006;33:718-29.

21. Ghandi TK, Graydon-Baker E, Huber CN, et al. Closing the loop: follow-up and feedback in a patient safety program. Jt Comm J Qual Patient Saf 2005;31:614-21.

22. Clark HR, Ramirez A, Drake $\mathrm{KN}$, et al. Utilization of an interorganizational network analysis to evaluate the development of community capacity among a community-academic partnership. Prog Community Health Partnersh 2014;8:41-51.

23. Provan KG, Veazie MA, Staten LK, et al. The use of network analysis to strengthen community partnerships. Public Adm Rev 2005;65:603-12.

24. Yessis J, Riley B, Stockton L, et al. Interorganizational relationships in the Heart and Stroke Foundation's Spark Together for Healthy Kids ${ }^{\mathrm{TM}}$ : Insights from using network analysis. Health Educ Behav 2013;40:435-505.

25. Fuller J, Oster C, Dawson S, et al. Improving the network management of integrated primary mental healthcare for older people in a rural Australian region: protocol for a mixed methods case study. BMJ Open 2014;4:e006304

26. Australian Government. My Region. Adelaide Hills, Fleurieu and Kangaroo Island. Australian Government; 5 Nov 2014 [cited 10 Feb 2015]. http://myregion.gov.au/south-australia/ adelaide-hills-fleurieu-kangaroo-island.aspx
27. Fried BJ, Johnsen MC, Starrett BE, et al. An empirical assessment of rural community support networks for individuals with severe mental disorders. Community Ment Health J 1998;34:39-56.

28. Sheaff $R$, Benson $L$, Farbus $L$, et al. Network resilience in the face of health system reform. Soc Sci Med 2010;70:779-86.

29. Ucinet 6 for Windows: Software for Social Network Analysis [program]. Harvard, MA: Analytic Technologies, 2002.

30. Strivastava A, Thomson S. Framework analysis: a qualitative methodology for applied policy research. J Admin Governance 2009;4:72-9.

31. Huxham C, Vangen S. Managing to collaborate: the theory and practice of collaborative advantage. New York: Routledge, 2005.

32. Breton M, Pineault R, Lavesque J-F, et al. Reforming healthcare systems on a locally integrated basis: is there a potential for increasing collaborations in primary healthcare? BMC Health Serv Res 2013;13:262.

33. Murayama H, Yamaguchi T, Nagata S, et al. The effects of an intervention program for promoting interorganizational network building between multidisciplinary agencies and community-based organizations: a cluster trial in Japan. BMC Public Health 2012;12:178.

34. Milward HB, Provan KG. A manager's guide to choosing and using collaborative networks. IBM Center for The Business of Government, 2006.

35. VicHealth. The Partnerships Analysis Tool. VicHealth [cited 25 Aug 2014]. https://www.vichealth.vic.gov.au/media-and-resources/ publications/the-partnerships-analysis-tool

36. Hardy B, Hudson B, Waddington E. What makes a good partnership? A partnership assessment tool. Leeds: Nuffield Institute for Health, Community Care Division, 2000.

37. Watson J, Speller V, Markwell S, et al. The Verona Benchmark: applying evidence to improve the quality of partnership. Promot Educ 2000;7:16-23.

38. Crotty M, Henderson J, Fuller J. Helping and hindering: perceptions of enablers and barriers to collaboration within a rural South Australian mental health network. Aust J Rural Health 2012;20:213-18.

39. Prell C, Hubacek K, Reed M. Stakeholder analysis and social network analysis in natural resource management. Soc Nat Resour 2009;22:501-18.

40. McKinn S, Bonner C, Jansen J, et al. Recruiting general practitioners as participants for qualitative and experimental primary care studies in Australia. Aust J Prim Health 2014;21:354-9.

41. National Voices. Principles for Integrated Care [internet]. National Voices [cited 10 Feb 2015]. http://www.nationalvoices.org.uk/sites/ www.nationalvoices.org.uk/files/principles for integrated care 20111021.pdf

42. Redding D. The narrative for person-centred coordinated care. J Integr Care 2013;21:315-25. 